\title{
spcadjust: An R Package for Adjusting for Estimation Error in Control Charts
}

\author{
by Axel Gandy and Jan Terje Kvaløy
}

\begin{abstract}
In practical applications of control charts the in-control state and the corresponding chart parameters are usually estimated based on some past in-control data. The estimation error then needs to be accounted for. In this paper we present an $\mathrm{R}$ package, spcadjust, which implements a bootstrap based method for adjusting monitoring schemes to take into account the estimation error. By bootstrapping the past data this method guarantees, with a certain probability, a conditional performance of the chart. In spcadjust the method is implement for various types of Shewhart, CUSUM and EWMA charts, various performance criteria, and both parametric and non-parametric bootstrap schemes. In addition to the basic charts, charts based on linear and logistic regression models for risk adjusted monitoring are included, and it is easy for the user to add further charts. Use of the package is demonstrated by examples.
\end{abstract}

\section{Introduction}

Control charts for statistical process monitoring are commonly used in a variety of different areas like industrial process control, medicine, finance, insurance, environmental science etc. See for instance Stoumbos et al. (2000), Woodall (2006), Frisén (2008), Schmid (2007a), and Schmid (2007b) for an overview.

A challenge in most practical applications of control charts is that the in-control state of the process to be monitored needs to be estimated. This introduces estimation error which needs to be accounted for. A common convention in many applications has been to assume the in-control distribution to be known and ignore the estimation error (e.g. Grigg and Farewell, 2004; Bottle and Aylin, 2008; Biswas and Kalbfleisch, 2008; Fouladirad et al., 2008; Gandy et al., 2010). However, there is an increasing awareness that the estimation error might have a detrimental effect on the performance of control charts and many authors have addressed this for various specific charts (e.g. Jones, 2002; Jones et al., 2004; Albers and Kallenberg, 2004, 2005; Albers et al., 2005; Jensen et al., 2006; Champ and JonesFarmer, 2007; Chatterjee and Qiu, 2009; Capizzi and Masarotto, 2009; Zhang et al., 2011; Saleh et al., 2015; Zhang et al., 2016).

Gandy and Kvaløy (2013) presented a bootstrap based method for adjusting for estimation error which applies to a wide variety of control charts and different performance measures. The method can in particular be used to give a guaranteed conditional in-control performance of the chart. A typical application is to calculate an adjusted signal limit of a chart to guarantee with high probability that the in-control average run length or hitting probability is not below/above a specified value. Theoretical properties and conditions needed for the method to apply are worked out in Gandy and Kvaløy (2013). A similar bootstrap approach was also briefly mentioned in Jones and Steiner (2012) in a study of risk-adjusted CUSUM charts.

We have developed an R package called spcadjust (Gandy and Kvaløy, 2016) which implements the bootstrap method for a number of different charts, with different performance measures and both parametric and non-parametric bootstrapping procedures. The package covers the most common set-ups for Shewhart, CUSUM and EWMA charts, including risk-adjusted charts. Moreover, it is easy for the user to add further charts, data models and estimation procedures.

There exist several other $\mathrm{R}$ packages for control charts. The surveillance package (Salmon et al., 2016) provides a variety of methods for monitoring, simulation and visualization of temporal and spatio-temporal data. The packages spc (Knot, 2016), qcc (Scrucca, 2004), IQCC (Scrucca, 2014) and qcr (Flores, 2016) provide functions for calculating various performance measures, signal limits, graphical displays etc. for a selection of classical control charts. The edcc package (Zhu and Park, 2013) has functions for economic design of control charts, while the MSQC package (Santos-Fernández, 2013) provides a tool kit for multivariate process monitoring. However, none of these packages include methods for taking into account the impact of estimation error on the performance of the charts, which is the main novelty of the spcadjust package.

In the next section, we give a brief introduction to the problem and describe the bootstrap approach for adjusting for estimation error. In the two sections thereafter we first illustrate basic use of the spcadjust package, then we describe details of the package and illustrate more advanced use. In the last section, a real data example is provided.

Stable versions of the package spcadjust are available on CRAN. Development versions are available in an open git repository (https://bitbucket.org/agandy/spcadjust/). We welcome con- 
tributions to the functionality of the package.

\section{Adjusting for estimation error in control charts}

Control charts are a set of statistical techniques for monitoring a stream of data over time. A typical application is to monitor whether a stream of measurements follows a certain distribution, often called the in-control distribution, over time. If the distribution of the measurements deviates from the in-control distribution in a certain way the control chart should quickly detect this. In more advanced situations, regression adjustments are needed, and the monitoring is based on detecting deviations from the regression model.

In most common control charts like Shewhart, CUSUM and EWMA charts, a function of the observations is plotted for each new observation and a signal is given if this function crosses a certain threshold. Both the parameters of the function and the threshold are in most cases calculated according to estimates of the in-control distribution. This implies that estimation error will affect the performance of the control charts. For instance, measurement errors might lead to control charts that give false alarms far too often.

We first give a motivating example, and then our bootstrap method for handling estimation error is described.

\section{Motivating example}

We consider an example with a CUSUM chart for monitoring changes in the mean of a stream of normally distributed data. Assume first that we know that the stream consists of independent observations $X_{1}, X_{2}, \ldots$ following a $N(\mu, \sigma)$ distribution in the in-control situation, and that we want to quickly detect if there is a change in the mean to the out-of-control situation $\mu+\Delta$. A standard CUSUM chart for this would be to plot $S_{t}$ versus $t$ where

$$
S_{t}=\max \left(0, S_{t-1}+\frac{X_{t}-\mu-\Delta / 2}{\sigma}\right), \quad S_{0}=0,
$$

where the chart signals once $S_{t}>c$. The threshold $c$ is calculated to give a certain performance of the chart if no change occurs. Often, $c$ is chosen to give a pre-specified in-control mean number of steps until a false alarm (often called average run length, ARL). Thus the CUSUM chart and the threshold $c$ should be calculated using the true $N(\mu, \sigma)$ in-control distribution.

However, in practice, the true in-control distribution $N(\mu, \sigma)$ is usually unknown, but estimated based on $n$ past in-control observations $X_{-n}, \ldots, X_{-1}$. For example, we can estimate the in-control mean by $\hat{\mu}=\frac{1}{n} \sum_{i=-n}^{-1} X_{i}$ and the in-control variance by $\hat{\sigma}^{2}=\frac{1}{n-1} \sum_{i=-n}^{-1}\left(X_{i}-\hat{\mu}\right)^{2}$. The CUSUM chart is then given by

$$
S_{t}=\max \left(0, S_{t-1}+\frac{X_{t}-\hat{\mu}-\Delta / 2}{\hat{\sigma}}\right), \quad S_{0}=0,
$$

which signals when $S_{t}>\hat{c}$ where $\hat{c}$ is calculated using the estimated in-control distribution $N(\hat{\mu}, \hat{\sigma})$.

Since the future in-control data come from the true in-control distribution $N(\mu, \sigma)$ rather than the estimated distribution $N(\hat{\mu}, \hat{\sigma})$, the performance of the chart might be substantially wrong, leading to more (or less) false alarms than expected.

A typical application of our bootstrap method described in the next subsections will be to calculate an adjustment to the naive threshold estimate $\hat{c}$ such that a certain in control behavior of the chart can be guaranteed with high probability.

As an illustration we generated $n=100$ past data from a $N(0,1)$ distribution giving estimates $\hat{\mu}=-0.028, \hat{\sigma}=0.921$. We want to monitor for an increase in the mean of the distribution by $\Delta=1$ and achieve an in-control ARL of 500.

Assuming (incorrectly) that the estimated parameters are the true parameters this would lead to a threshold of $\hat{c}=4.1$. Our bootstrap method leads to an adjusted threshold of 5.5, calculated such that the ARL of 500 is achieved with a probability of $90 \%$.

The result of running the CUSUM chart with these estimated parameters on a stream of $N(0,1)$ in-control data is illustrated in the top row of Figure 1. There is a false alarm with the unadjusted threshold, but not with the adjusted threshold.

In the bottom row of Figure 1 the same CUSUM chart is run on data which are out of control from observation 81 and onwards. There is only a slight delay in the detection of the out-of-control situation with the adjusted threshold. Also, there are two false alarms in the first 80 observations with the unadjusted threshold. 

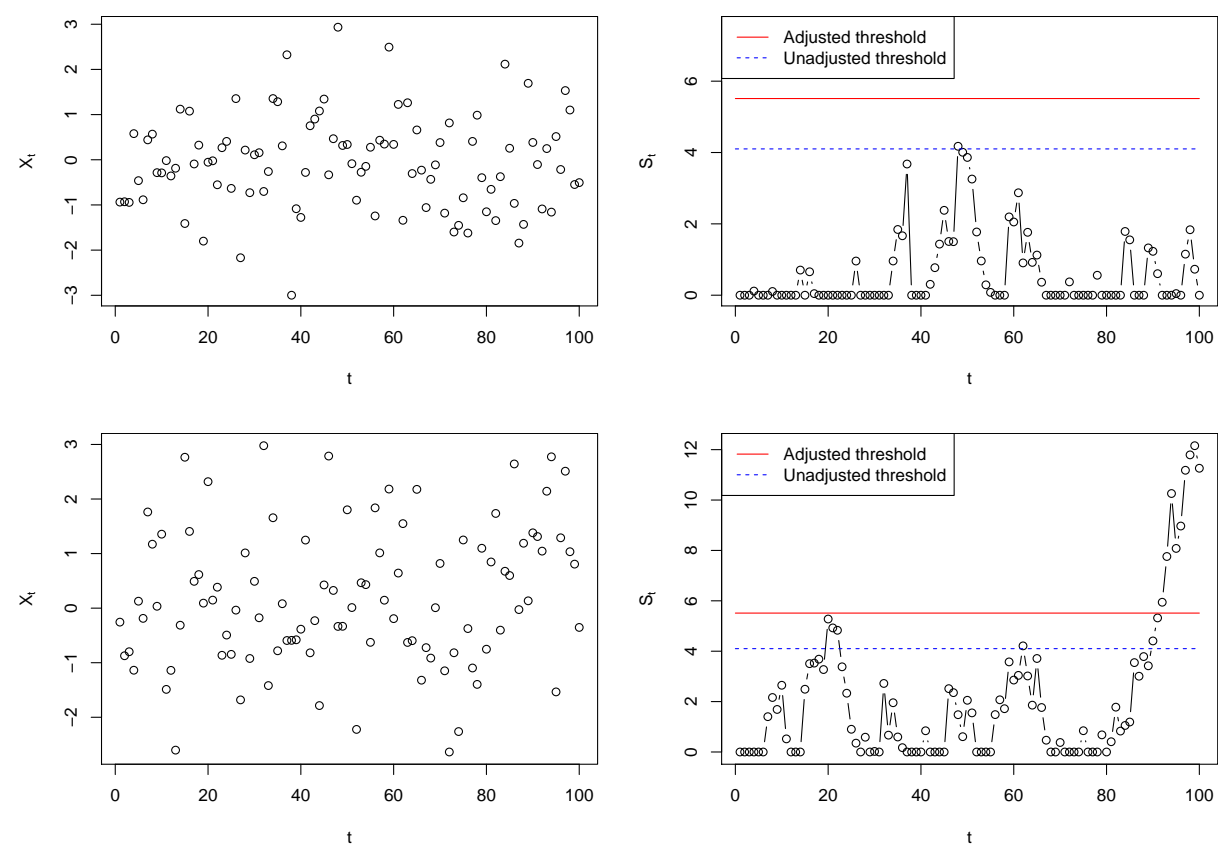

Figure 1: CUSUM charts for the motivating example. Top row: in-control data. Bottom row: data which switch to out-of-control from observation 81. The left figures show the observed data streams, the right the resulting CUSUM charts which were run with the estimated parameters. Adjusted and unadjusted thresholds are indicated.

\section{Homogeneous observations}

In this section we describe our bootstrap procedure for situations with homogeneous observations. Assume that in-control we have a stream of independent observations $X_{1}, X_{2}, \ldots$, following a distribution $P$. A control chart is used to detect when observations are no longer coming from $P$, also called an out of control situation, for instance a shift in mean or variance.

To run such a chart, certain parameters, $\xi$, calculated from $P$ are usually needed. However, in most applications the exact distribution $P$ is unknown, we only have an estimate of the distribution, and thus have to run the chart with estimated parameters. In the example in the previous section, the parameters needed to run the chart are $\xi=(\mu, \sigma)$ and the unknown in-control distribution $P$ is $N(\mu, \sigma)$.

Common performance measures for control charts are the ARL and the hitting probability of the chart within a certain number of steps. These depend both on the unknown $P$ as well as on the parameters $\xi$. Indeed, let $\tau$ denote the time (observation number) at which a chart gives a signal, e.g. the first time a CUSUM chart has a value above $c$. The distribution of this stopping time $\tau$ depends on $P$ and $\xi$. We can express the ARL as $A R L(P ; \xi)=\mathbb{E}(\tau(\xi))$, where the expectation is with respect to $P$. The probability of signaling within $m$ time steps (for some finite $m>0$ ) can be expressed as $\operatorname{hit}(P ; \xi)=P(\tau(\xi) \leq m)$.

The signal limit $c$ is chosen to achieve a certain in-control performance of the chart. For charts which signal when a threshold $c$ is crossed we can express this as follows.

- $c_{A R L}(P ; \xi)=\inf \{c>0: A R L(P ; \xi) \geq \gamma\}$ for some $\gamma>0$, i.e. the threshold needed to give an in-control $A R L$ of $\gamma$.

- $c_{\text {hit }}(P ; \xi)=\inf \{c>0: \operatorname{hit}(P ; \xi) \leq \beta\}$ for some $0<\beta<1$, which is the threshold needed to give a false alarm probability of $\beta$.

Again, both $c_{A R L}$ and $c_{\text {hit }}$ depend on $P$ and $\xi$.

Let $\hat{P}$ and $\hat{\zeta}=\xi(\hat{P})$ denote the estimated distribution and estimated parameters, estimated from past in-control data $X_{-n}, \ldots, X_{-1}$. Since we have to run the chart with $\hat{\xi}$ while future in-control data follow $P$ ideally we should use the threshold $c_{A R L}(P ; \hat{\xi})$ or $c_{h i t}(P ; \hat{\xi})$. These are unknown since $P$ is unknown. Instead in practice $c_{A R L}(\hat{P} ; \hat{\xi})$ or $c_{h i t}(\hat{P} ; \hat{\xi})$ is often used, but this may lead to performance substantially off from the nominal performance.

The suggestion in Gandy and Kvaløy (2013) is to calculate, by bootstrapping, an adjusted threshold which with high probability will guarantee that the in-control performance is not worse than the nominal value. For instance to calculate an adjustment to $c_{A R L}(\hat{P} ; \hat{\xi})$ which with a probability $1-\alpha$ 
guarantees that the true in-control $A R L$ is at least as large as the nominal value.

To make a unified presentation of the bootstrap we let $q$ be a common notation for quantities of interest like $c_{A R L}$ and $c_{\text {hit }}$, or simple transformations such as $\log \left(c_{A R L}\right)$ and $\log \left(c_{\text {hit }}\right)$. Further let $p_{\alpha}$ be a constant such that

$$
P\left(q(\hat{P} ; \hat{\xi})-q(P ; \hat{\zeta})>p_{\alpha}\right)=1-\alpha,
$$

which implies the following bound on the quantity of interest

$$
P\left(q(P ; \hat{\xi})<q(\hat{P} ; \hat{\xi})-p_{\alpha}\right)=1-\alpha .
$$

Since $P$ is unknown we cannot calculate $p_{\alpha}$, but an approximation can be obtain by bootstrapping. Let $\hat{P}^{*}$ denote a parametric or non-parametric bootstrap replicate of the estimated in-control distribution $\hat{P}$, based on the same sample size $n$ as $\hat{P}$, and let $\hat{\zeta}^{*}=\xi\left(\hat{P}^{*}\right)$. Then we can approximate $p_{\alpha}$ by $p_{\alpha}^{*}$ where

$$
P\left(q\left(\hat{P}^{*} ; \hat{\xi}^{*}\right)-q\left(\hat{P} ; \hat{\zeta}^{*}\right)>p_{\alpha}^{*} \mid \hat{P}\right)=1-\alpha .
$$

Then an approximate upper bound which guarantees a certain performance with an approximate probability of $1-\alpha$ is $q(\hat{P} ; \hat{\xi})-p_{\alpha}^{*}$. We can also think of

$$
\left(-\infty, q(\hat{P} ; \hat{\xi})-p_{\alpha}^{*}\right)
$$

as a sort of one-sided (approximate) confidence interval for $q(P ; \hat{\xi})$. The bootstrap distribution has to be approximated by simulations, easily performed by generating $B$ bootstrap samples from $\hat{P}$ and calculating $q\left(\hat{P}^{*} ; \hat{\xi}^{*}\right)-q\left(\hat{P} ; \hat{\xi}^{*}\right)$ for each bootstrap sample. For further details, including theoretical properties, we refer to Gandy and Kvaløy (2013).

If we return to the example in the previous section and consider the threshold needed to get a certain in-control $A R L$ value $\gamma$, we adapt the above procedure with either $q=c_{A R L}$ or $q=$ $\log \left(c_{A R L}\right)$. The latter is recommended as the log-transform usually improves the precision (Gandy and Kvaløy, 2013). Then $B$ bootstrap samples of size $n$ are generated from $\hat{P}$ and from these we calculate $\hat{P}_{1}^{*}, \ldots, \hat{P}_{B}^{*}$ and $\hat{\xi}_{1}^{*}, \ldots, \hat{\zeta}_{B}^{*}$. With $q=\log \left(c_{A R L}\right)$ we then calculate $p_{\alpha}^{*}$ as the $1-\alpha$ empirical quantile of $\log c_{A R L}\left(\hat{P}_{b}^{*} ; \hat{\xi}_{b}^{*}\right)-\log c_{A R L}\left(\hat{P} ; \hat{\xi}_{b}^{*}\right), b=1, \ldots, B$, and obtain the adjusted threshold $\exp \left(\log c_{A R L}(\hat{P} ; \hat{\xi})-p_{\alpha}^{*}\right)=c_{A R L}(\hat{P} ; \hat{\xi}) \exp \left(-p_{\alpha}^{*}\right)$. With this adjusted threshold there is an approximate $1-\alpha$ probability that the actual $A R L$ of the charts is at least $\gamma$.

\section{Risk-adjusted charts}

In many applications of control charts the units being monitored are heterogeneous, for instance when monitoring data from human beings. To make reasonable monitoring systems in such situations the explainable part of the difference between units should be accounted for by regression models. Charts based on regression models are often called risk-adjusted, an overview of some such charts is found in Grigg and Farewell (2004).

For risk-adjusted charts the regression model has to be estimated based on past data, and the impact of estimation error thus has to be taken into account. The bootstrap procedure outlined in the previous section also applies to risk adjusted charts. Let the stream of observations now be denoted $\left(Y_{1}, X_{1}\right),\left(Y_{2}, X_{2}\right), \ldots$, where $Y_{i}$ is a response variable and $X_{i}$ a corresponding vector of covariates. Further let $P$ denote the joint distribution of $\left(Y_{i}, X_{i}\right)$. For regression models we recommend to use a non-parametric bootstrap. Let $\hat{P}$ be the empirical distribution which puts weight $1 / n$ on each of the $n$ past observations $\left(Y_{-n}, X_{-n}\right), \ldots,\left(Y_{-1}, X_{-1}\right)$. Then by resampling from this $\hat{P}$ the bootstrap procedure also applies to a wide variety of risk adjusted charts (Gandy and Kvaløy, 2013).

As an example consider a CUSUM chart for a linear regression model. Suppose that in-control $\mathbb{E}\left(Y_{i} \mid X_{i}\right)=X_{i} \beta$ (where the first component of $X_{i}$ is 1 ) and we want to detect a change in the mean response to $\mathbb{E}\left(Y_{i} \mid X_{i}\right)=\Delta+X_{i} \beta$ for some $\Delta>0$. For linear regression models it is natural to base the monitoring on the residuals of the model (Horváth et al., 2004). A CUSUM to monitor changes in the mean can for instance be defined by

$$
S_{t}=\max \left(0, S_{t-1}+Y_{t}-X_{t} \beta-\Delta / 2\right), \quad S_{0}=0,
$$

which signals when $S_{t} \geq c$. In practice since $\beta$ and the distribution of the residuals, $P_{\epsilon}$, are estimated the CUSUM is run with $\hat{\beta}$ and a threshold calculated e.g. as $c_{A R L}\left(\hat{P}_{\epsilon} ; \hat{\beta}\right)$. This might lead to an ARL far off from the nominal (Gandy and Kvaløy, 2013).

To account for the estimation error we can use the bootstrap procedure from the previous section with e.g. $q=\log \left(c_{A R L}\right)$ and non-parametric bootstrapping as outlined above to calculate the adjusted threshold $\exp \left(\log c_{A R L}\left(\hat{P}_{\epsilon} ; \hat{\beta}\right)-p_{\alpha}^{*}\right)=c_{A R L}\left(\hat{P}_{\epsilon} ; \hat{\beta}\right) \exp \left(-p_{\alpha}^{*}\right)$. Using this adjusted threshold there 


\begin{tabular}{ll}
\hline class name & data model \\
\hline "SPCModelNormal" & $\begin{array}{l}\text { normally distributed updates of the form }\left(X_{t}-\mu-\Delta / 2\right) / \sigma \\
\text { (Sections CUSUM chart with estimated in-control state and }\end{array}$ \\
"SPCModelNonparCenterScale" & $\begin{array}{l}\text { Shewhart chart with estimated in-control state) } \\
\text { updates }\left(X_{t}-\mu-\Delta / 2\right) / \sigma, \text { no distributional assumptions } \\
\text { (Section CUSUM chart with estimated in-control state) }\end{array}$ \\
"SPCModelNonpar" & user defined updates, no distributional assumptions \\
"SPCModellm" & $\begin{array}{l}\text { linear regression model with updates } Y_{t}-X_{t} \beta-\Delta / 2 \\
\text { (Sections CUSUM chart with linear regression model and }\end{array}$ \\
"SPCModellogregLikRatio" & EWMA chart with linear regression model) \\
& $\begin{array}{l}\text { logistic regression model, likelihood ratio updates } \\
\text { (Section Application to cardiac surgery data) } \\
\text { "SPCModellogregOE" }\end{array}$ \\
& logistic regression model, observed minus expected \\
& updates \\
\hline
\end{tabular}

Table 1: Overview of pre-implemented data models.

is an approximate $1-\alpha$ probability that the actual $A R L$ of the charts is at least as large as desired. Further risk-adjusted charts are discussed in Section EWMA chart with linear regression model and Section Application to cardiac surgery data.

\section{Other adjustments}

So far we have focused on how to adjust the signal limit of the control chart to achieve a certain performance with a high probability. In practice that will be a typical application, but it is easy to change focus to other quantities. For instance, instead of adjusting the threshold to obtain a certain ARL we could instead fix the threshold and calculate which ARL we with high probability at least will achieve. For instance with Shewhart charts it is very common to use $c=3$ standard deviations as signal limit, and if we with estimated parameters still choose to stick with this limit we can use the bootstrap approach to calculate a lower limit of the achieved ARL. In practice this is done by defining the appropriate $q$-function and then run the general bootstrap procedure as before. For the Shewhart example with fixed $c$ and focus on $A R L$ the $q$ would simply be the $A R L$ or $\log (A R L)$. See Gandy and Kvaløy (2013) for details and Section Shewhart chart with estimated in-control state for an example.

Another variant which we consider further in Section Implementing a new type of chart is Shewhart charts with non-symmetric signal limits for skew distributions. For such charts the signal limit can be defined in terms of the quantiles corresponding to a certain tail probability $\alpha$. Then we can via the bootstrap find the appropriate adjustment of this $\alpha$ to achieve a certain performance with high probability.

\section{Basic usage of the package}

In this section we discuss how the package can be used for pre-implemented chart types and data models. The framework provided in the package can also easily be extended to work with other charts, data models and/or estimation procedures as will be explained in Section Details of the package and advanced usage.

An important basic structure of the package is that chart types and data models are implemented in separate objects and in such a way that they can be flexibly combined. The implemented chart types are the Shewhart chart (SPCShew), the CUSUM chart (SPCCUSUM) and the EWMA chart (SPCEWMA). Table 1 lists the implemented data models.

Calculation of charts and chart properties like thresholds, ARLs and hitting probabilities are defined with the chart type object. Estimation of chart parameters, the form and cdf of updates and the bootstrap procedure are defined in the data model object. With updates we mean the quantity added to the chart in each step. Parametric bootstraping is used in the normal model, nonparametric bootstraping in all the other pre-implemented models.

The parameter $\Delta$ in the updates in some of the data models listed in Table 1 specifies the out-ofcontrol situation for CUSUM charts, but should be set to the default value Delta $=0$ for Shewhart and EWMA charts.

The main steps for basic usage of the package is to define a chart object with the new() function and to calculate the properties of interest with the SPCproperty() function. A generic description of 
these functions is given below.

To define a chart object we need to specify the combination of chart type and data model as follows: chartgeneric <- new ("Charttype", model = Datamodel $($ Delta $=x), \ldots$ )

Here "Charttype" should be one of "SPCShew", "SPCCUSUM" or "SPCEWMA" and "Datamodel" should be one of the class names listed in Table 1. Finally $x$ should be 0 for Shewhart and EWMA chart and set to the desired value for CUSUM charts.

The following function invokes the bootstrap procedure and calculate the property of interest for the chart:

SPCproperty (data, nrep, property = "specifyproperty", chart = chartgeneric,
params $=$ list (specifyparameters), covprob=0.9, parallel $=1, \ldots$ )

Here data are the past observations (usually a vector) and nrep is the number of bootstrap replications. Further, specifyproperty specifies the property of interest, with choices calARL, calhitprob, ARL and hitprob. The two first choices calculate a calibrated threshold to achieve, with high probability, a desired ARL or a desired hitting probability. Based on a specified choice of threshold, the two last choices calculate the smallest ARL or the largest hitting probability that is attained with high probability. Necessary parameters are given in specifyparameters (depending on the property this includes the desired ARL, the desired hitting probability or the threshold). Finally, covprob, gives the desired coverage probability $1-\alpha$ with a default of $90 \%$. The bootstrap can be sped up by parallel processing by specifying the number of cores to be used via the 'parallel' option.

Further functions and details are explained in the examples below. In the two first subsections below we consider situations with homogeneous observations, in the next subsections, we consider situations with risk-adjusted charts.

\section{CUSUM chart with estimated in-control state}

We now return to the motivating example in Section Motivating example. Recall that we want to run a CUSUM chart of the form (1) to monitor for a change in the mean in a stream of normally distributed data. We first define the chart object by specifying chart type and data model.

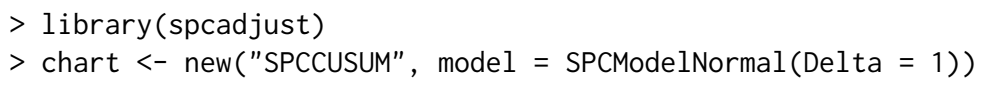

Here "SPCCUSUM" specifies that it should be a CUSUM chart, and SPCModelNormal (Delta = 1) specifies that it is a model with normally distributed updates of the form $\left(X_{t}-\mu-\Delta / 2\right) / \sigma$ with $\Delta=1$.

Next we generate $n=100$ past observations and use the function xiofdata to calculate the estimated chart parameters $\hat{\xi}$.

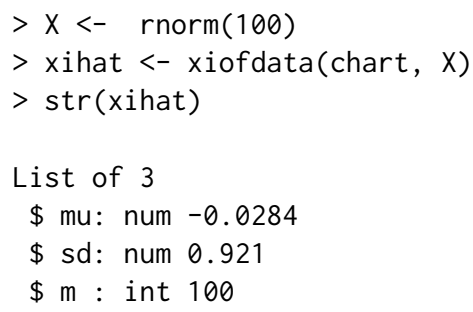

Now we can use the function SPCproperty() to calculate the naive and adjusted threshold:

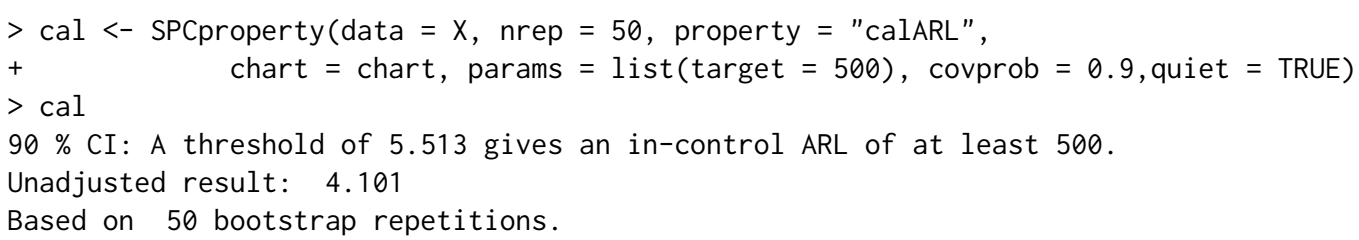

Here property = "calARL" specifies that the chart should be calibrated to achieve a certain ARL, this ARL is specified to be 500 (target $=500$ ) and the probability of attaining at least this ARL specified to be $90 \%$ (covprob $=0.9$ ). The adjusted threshold of 5.5 is calculated using parametric bootstrapping with nrep replications assuming normality of the observations. For real applications nrep should of course be more than 50. If nonparametric bootstrapping is preferred the model specification in the definition of the chart should be replaced by SPCModelNonparCenterScale().

If we rather would like to calibrate the chart according to a certain hitting probability, for instance a hitting probability of 0.05 within 100 steps, this is achieved by specifying property = "calhitprob" and params $=$ list $($ target $=0.05$, nsteps $=100)$ : 


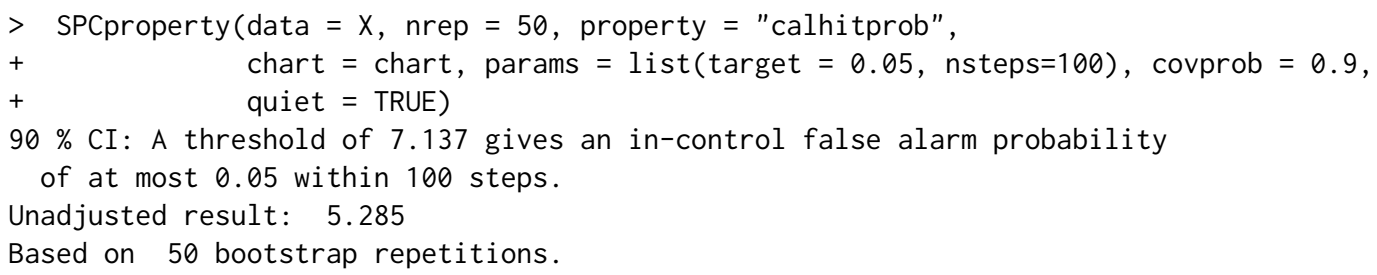

The function runchart is used to run the chart on future data, and the option xi specifies which parameters to use when running the chart:

$>$ newX $<-$ rnorm(100)

$>\mathrm{S}<-\operatorname{runchart}($ chart, newdata $=$ newX, $\mathrm{xi}=$ xihat $)$

The following code produces the plots in the first row of Figure 1, using the ARL calibrated threshold calculated above:

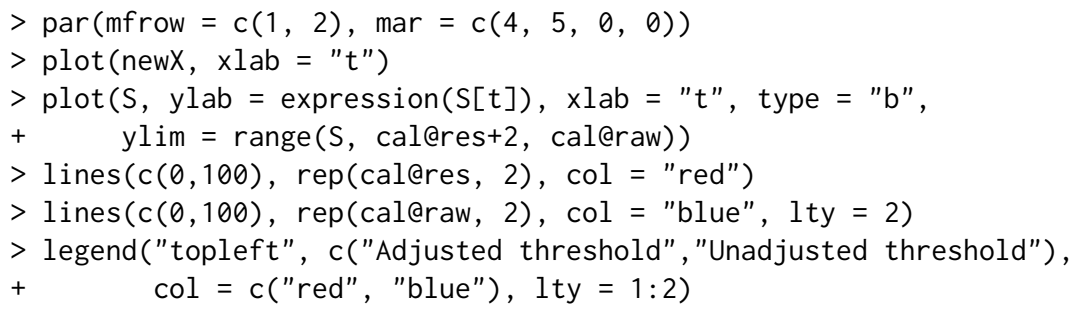

\section{Shewhart chart with estimated in-control state}

Next we consider a two-sided Shewhart chart, assuming that all observations are normally distributed. The in-control mean and standard deviation are estimated from $n$ past in-control observations $X_{-n}, \ldots, X_{-1}$. For new observations $X_{1}, X_{2}, \ldots$ a two-sided Shewhart chart is defined by

$$
S_{t}=\frac{X_{t}-\hat{\mu}}{\hat{\sigma}}
$$

which signals when $\left|S_{t}\right|>c$ for some threshold $c$. A common choice for Shewhart charts is to set $c=3$, corresponding to three standard deviations if the chart is run with the correct in-control mean and standard deviation.

We first define the chart by

$>$ chartShew <- new("SPCShew", model = SPCModelNormal(), twosided $=$ TRUE)

and then generate $n=250$ past observations and estimate the chart parameters:

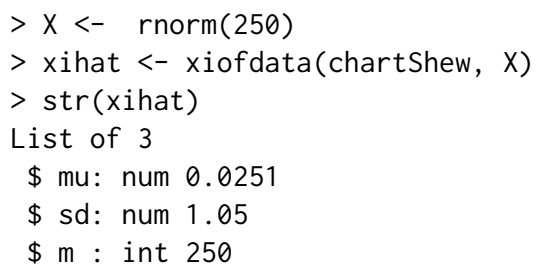

If the Shewhart chart is run with the standard threshold $c=3$, we can use the bootstrap method to calculate a lower limit for the actual ARL of the chart by specifying property = "ARL" and params = list (threshold $=3$ ):

$>$ SPCproperty (data $=X$, nrep $=50$, property $=$ "ARL", chart $=$ chartShew,

$+\quad$ params $=$ list $($ threshold $=3)$, quiet $=$ TRUE)

$90 \%$ CI: A threshold of 3 gives an in-control ARL of at least 213.1.

Unadjusted result: 370.4

Based on 50 bootstrap repetitions.

A two-sided Shewhart chart for normally distributed data with true parameters and a threshold of $c=3$ will correspond to an ARL of roughly 370 . We can thus compute an adjusted threshold that with roughly $90 \%$ probability results in an average run length of at least 370 in control:

$>$ cal <- SPCproperty $($ data $=X$, nrep $=50$, property $=$ "calARL", chart $=$ chartShew,

$+\quad$ params $=\operatorname{list}($ target $=370)$, quiet $=$ TRUE $)$ 


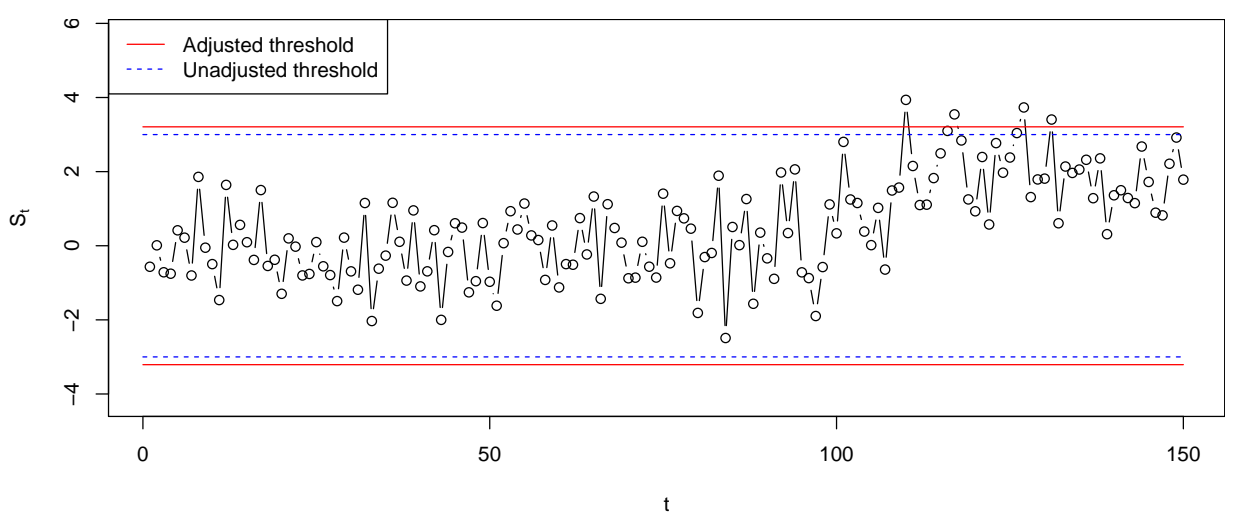

Figure 2: Shewhart charts with estimated parameters run on data which are in-control until observation 100 and with a shift in the mean from observation 101 an onwards.

$>\mathrm{cal}$

$90 \%$ CI: A threshold of 3.209 gives an in-control ARL of at least 370 .

Unadjusted result: 3

Based on 50 bootstrap repetitions.

Finally we run the chart with new observations. The simulated new observations are in-control for the first 100 observations, and then there is a shift in the mean from observations 101 an onwards. The corresponding plot is given in Figure 2.

$>$ newX $<-\operatorname{rnorm}(150$, mean $=c(\operatorname{rep}(0,100), \operatorname{rep}(2,50)))$

$>\mathrm{S}<-\operatorname{runchart}($ chartShew, newdata $=$ newX, $\mathrm{xi}=\mathrm{xihat})$

\section{CUSUM chart with linear regression model}

The set up is as described in Section Risk-adjusted charts, and with estimated regression coefficients $\hat{\beta}$ the CUSUM is

$$
S_{t}=\max \left(0, S_{t-1}+Y_{t}-X_{t} \hat{\beta}-\Delta / 2\right), \quad S_{0}=0 .
$$

The following generates a data set of past observations from the model $\mathbb{E}(Y)=2+x_{1}+x_{2}+x_{3}$ with standard normal noise and distribution of the covariate values as specified below.

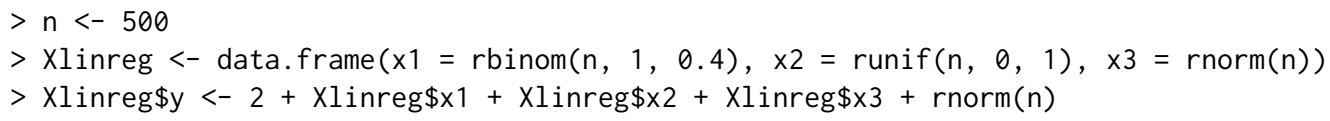

Next, we initialize the chart

$>$ chartlinregCUSUM <-

+ new("SPCCUSUM", model $=\operatorname{SPCModellm}\left(\right.$ Delta $=1$, formula $\left.=" y^{\sim} \times 1+x 2+x 3 "\right)$ )

where SPCModellm() uses non-parametric bootstrapping as explained in Section Risk-adjusted charts. The estimated parameters for running the chart, $\hat{\beta}$, are:

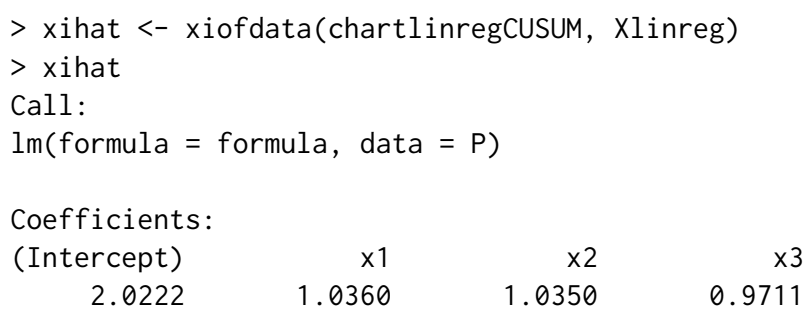

Next we find the threshold that with roughly $90 \%$ probability results in an average run length of at least 100 in control.

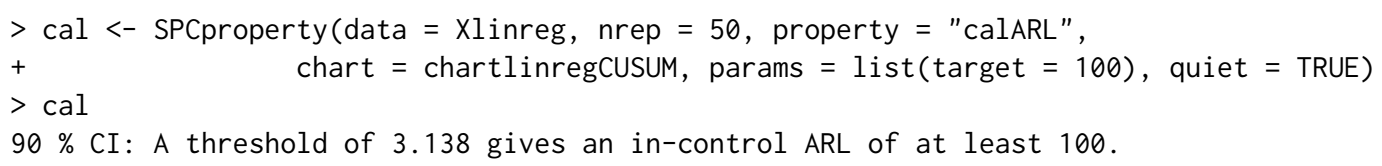




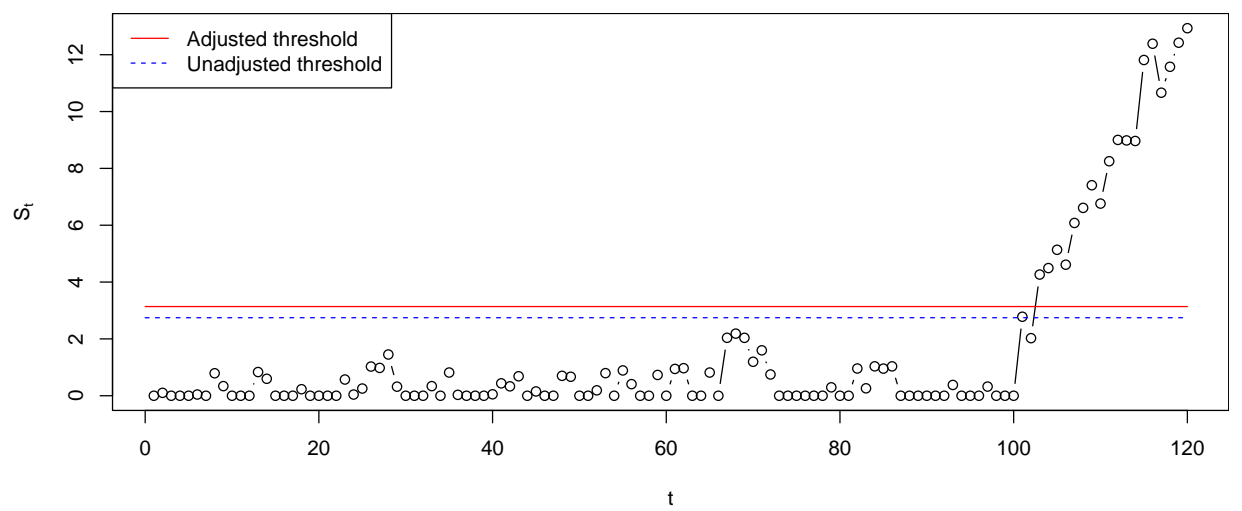

Figure 3: CUSUM chart for a linear regression model with estimated parameters. The data shift from in-control to out-of-control from observation 101 an onwards.

Unadjusted result: 2.745

Based on 50 bootstrap repetitions.

Finally, we run the chart with new observations that are in-control for the first 100 observations and then switches to out-of-control. A plot of the resulting CUSUM is given in Figure 3.

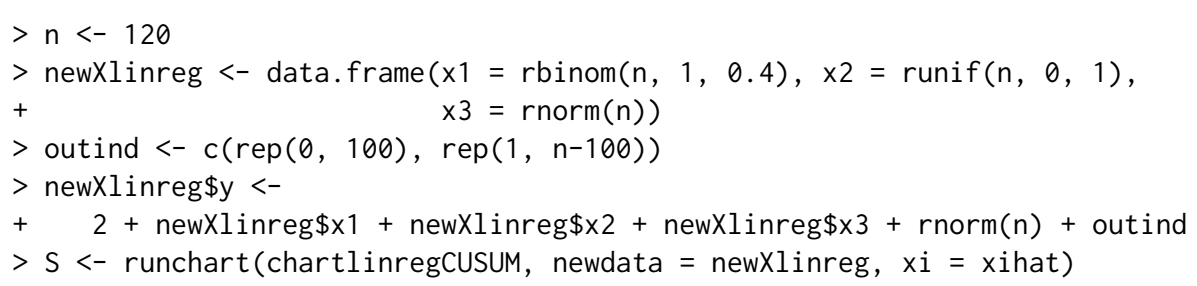

\section{EWMA chart with linear regression model}

An EWMA chart based on the residuals of a linear regression model can be defined by

$$
M_{t}=\lambda\left(Y_{t}-X_{t} \beta\right)+(1-\lambda) M_{t-1}, \quad M_{0}=0,
$$

where $\lambda$ is a smoothing parameter determining how to weight the most recent observation versus the past data. We can now set up the chart, calculate adjusted thresholds and run the chart on the new data with estimated parameters in the same manner as for the CUSUM chart. The only differences are that we have to specify "SPCEWMA", Delta $=0$ and a value of $\lambda$ when the chart is initialized.

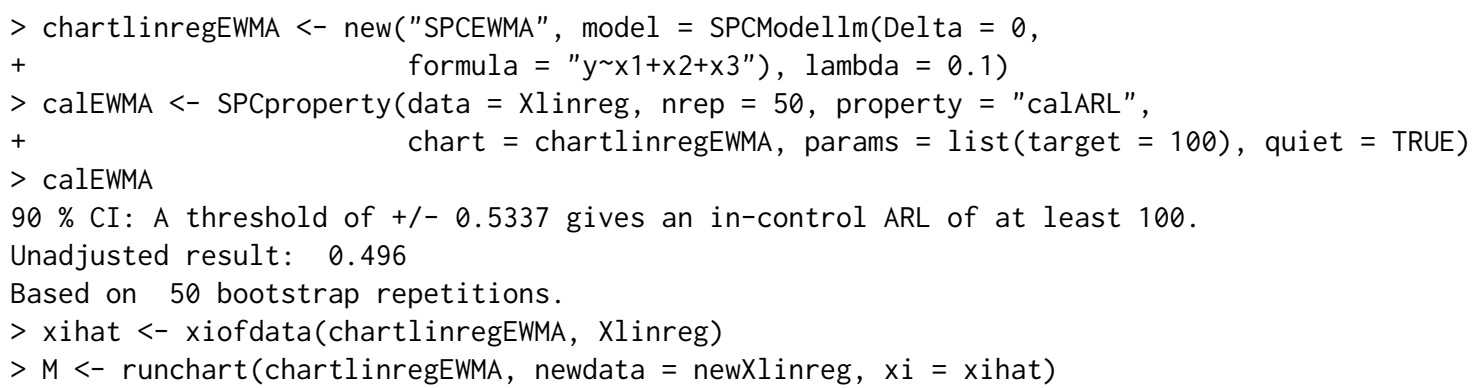

A plot of the resulting EWMA chart is given in Figure 4.

Further usage of risk-adjusted charts will be demonstrated in Section Application to cardiac surgery data where a CUSUM for logistic regression will be explained and used.

\section{Details of the package and advanced usage}

A basic structure of the package is, as explained in the introduction of Section Basic usage of the package, a definition of two types of objects. We will now look further into the details of these two objects and how they can be used to add new charts, new data models and other estimation procedures. 


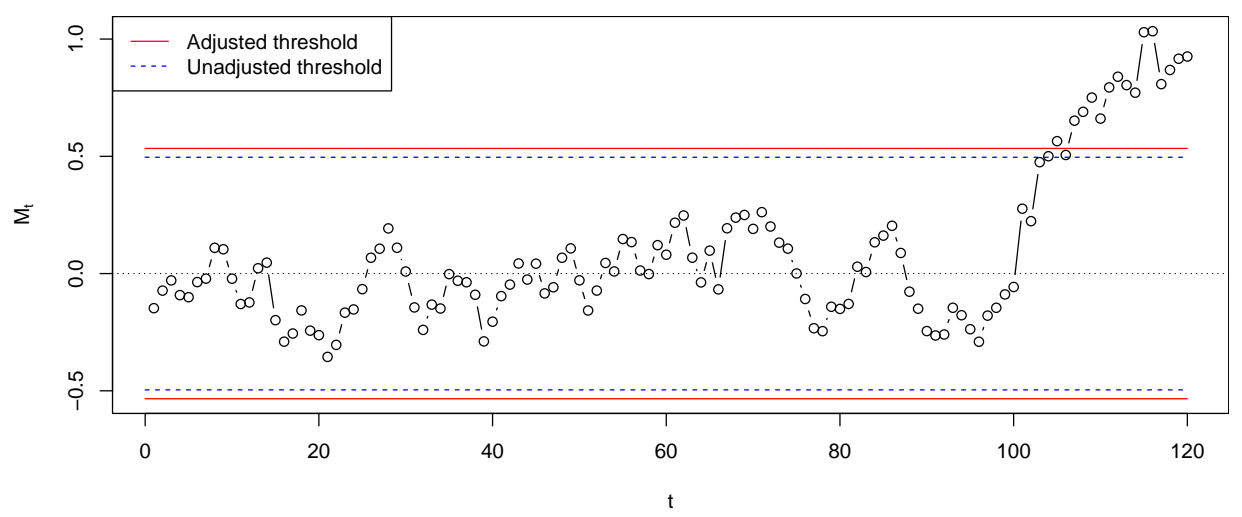

Figure 4: EWMA chart for a linear regression model with estimated parameters. The data shift from in-control to out-of-control from observation 101 an onwards.

One object is an S3 class of type "SPCDataModel" that implements how observed data are used to fit the model and how updates for the chart are being computed. The second object is an S4 class of type "SPCchart" which implements how these updates are converted into charts and how the charts are being calibrated. The main advantage of this separation into two different objects is that it reduces the amount of redundancy in the code.

The package was originally developed with S4 classes only, to take advantage of the more flexible method dispatch. However, to improve performance, the data model classes, whose methods are called very frequently, were switched to S3 classes.

\section{Bespoke data model}

We first focus on how to generate a bespoke data model. For this one needs to implement a class of type "SPCDataModel". Every element of the class has to consist of a list of the following functions: updates, getcdfupdates, Pofdata, resample, xiofP, which have to be of a specific form. The arguments generally have the following meaning: $x i$ denotes the parameter vector needed to create updates for running the chart from observed data, data is observed data, $\mathrm{P}$ is a data model.

- updates (xi,data): Returns updates for the chart using the parameter xi and the observed data data.

- Pofdata(data): Estimates a probability model from the data.

- $x$ iofP $(P)$ : Computes the parameter xi needed to compute updates from an (estimated) probability model P.

- resample(P): Generates a new data set from the probability model P.

- getcdfupdates $(P, x i)$ : Returns the cumulative distribution function (CDF) of updates with data generated from the probability model $P$ and updates computed using the parameter $x i$.

In the following we give some examples.

\section{Robust estimation}

Consider again the example discussed in Sections Motivating example and CUSUM chart with estimated in-control state of a CUSUM chart which assumes a normal distribution of the observations with unknown mean and standard deviation. We now demonstrate how to change the estimators to use the median the mean absolute deviation (MAD) instead of using the mean and the sample standard deviation. Using these robust estimators could be desirable if there could be outliers present in the past in-control data.

For this we only need to override one function of the existing data model "SPCModelNormal", the method Pofdata that estimates the parameters. The format of the input and output for the new Pofdata function needs to be unchanged. The following code first list the old function and then overrides it with the new.

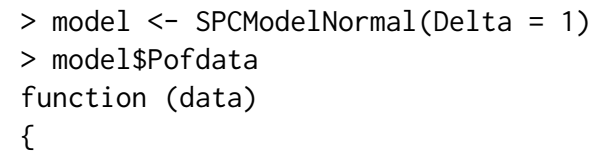




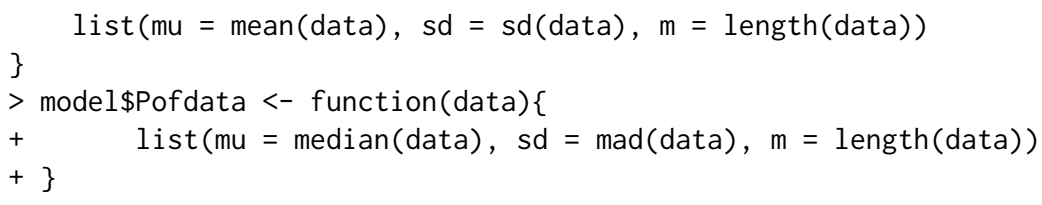

Properties of this chart can then be computed as before:

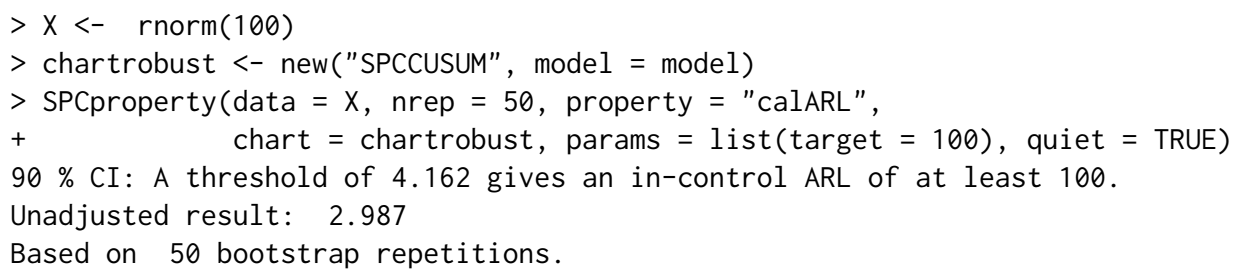

\section{Parametric exponential CUSUM chart}

In this example we illustrate how to construct a CUSUM chart that assumes that the observations are coming from an exponential distribution with unknown rate $\lambda$ in control. Again, only the data model needs to be defined, but now all functions are needed from scratch. I.e. we need to define all the functions updates, Pofdata, XiofP, resample and getcdfupdates. The basic CUSUM chart class "SPCCUSUM" will be used without changes.

The updates for a CUSUM chart can in general situations be based on the log likelihood ratio between an out-of-control model and the in-control model (Hawkins and Olwell, 1998). I.e. the CUSUM can be written

$$
S_{t}=\max \left(0, S_{t-1}+R_{t}\right), \quad S_{0}=0,
$$

where the update $R_{t}$ is the log likelihood ratio for observation $t$. Suppose that we want to detect a change of the rate to $\lambda \Delta$ for some given $\Delta>0, \Delta \neq 1$. To define the updates, we need to compute the log likelihood ratio between the out-of-control and the in-control model for an observation $X_{t}$, which gives

defining the function updates.

$$
R_{t}=\log \left(\frac{\lambda \Delta \exp \left(-\lambda \Delta X_{t}\right)}{\lambda \exp \left(-\lambda X_{t}\right)}\right)=\log (\Delta)-\lambda(\Delta-1) X_{t}
$$

To define the data model the CDF of these updates must also be computed. This can be done in closed form, but requires distinguishing the case $\Delta>1$ and $\Delta<1$ and taking into account that the rate parameter used in the updates typically differs from the true rate parameter. Let $\hat{\lambda}$ be the rate parameter used in the updates (typically an estimated parameter) and $\lambda$ be the true parameter. Then the cdf (conditional on the value of $\hat{\lambda}$ ) is

$$
P\left(\log (\Delta)-\hat{\lambda}(\Delta-1) X_{i} \leq x\right)= \begin{cases}1-\exp (-\lambda(x-\log (\Delta)) /(\hat{\lambda}(1-\Delta)) & \text { for } \Delta<1 \\ \exp (-\lambda(\log (\Delta)-x) /(\hat{\lambda}(\Delta-1)) & \text { for } \Delta>1\end{cases}
$$

which needs to be implemented in getcdfupdates. We decide to use parametric resampling of the data (resample) and we decide to estimate the parameter $\lambda$ based on the past observations $X_{-n}, \ldots, X_{-1}$ using the maximum likelihood estimator $\hat{\lambda}=n / \sum_{i=1}^{n} X_{-i}$.

The following code implements this.

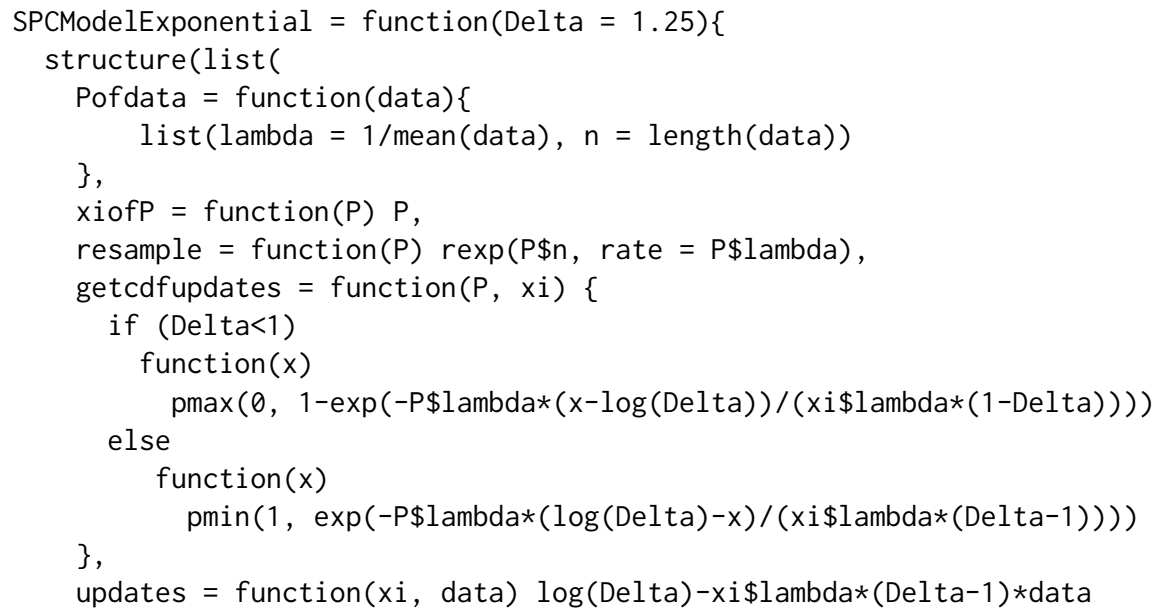


Next, we put this into practice. First we initiate the chart.

$>$ ExpCUSUMchart <- new("SPCCUSUM", model = SPCModelExponential (Delta $=1.25)$ )

The following creates some past observations and compute the threshold needed to achieve an ARL of 1000.

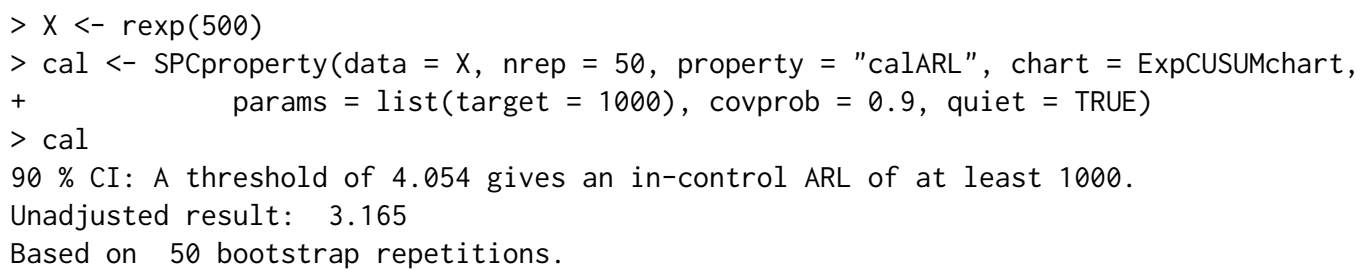

Finally, we generate some new data and make a CUSUM plot with thresholds which is displayed in Figure 5.

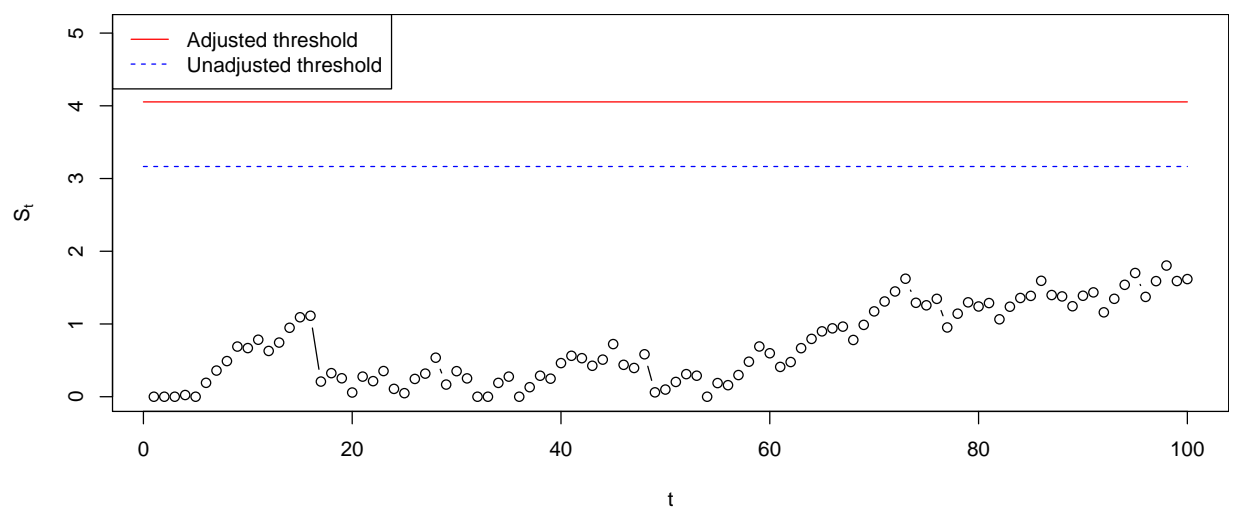

Figure 5: CUSUM chart for the rate of an exponential distribution with estimated parameters. The data are in control.

\section{Implementing a new type of chart}

We now discuss the chart model and how to implement new charts. Every chart is an S4 class derived from the class "SPCchart". It has one slot, model, which contains the data model to be used with the chart. The main method that needs to be implemented is the method getq, which computes desired properties of a given control chart. It receives two arguments: which property to report (a string, e.g. ARL, hi tprob, calARL, calhi tprob) and additional parameters for this property, e.g. a threshold when computing the ARL (property ARL), a threshold and a number of steps when computing hitting probabilities (property hitprob), a desired ARL when calibrating the threshold (property calARL).

We now give one example of how to implement a new chart. Suppose the in-control distribution is assumed to have a distribution with continuous $\operatorname{cdf} F$, which does not need to be symmetric. Then we can define a Shewhart type chart which signals if an observation $X$ is in the upper $\alpha / 2$ or lower $\alpha / 2$ quantile for a given threshold $\alpha>0$, i.e. if

$$
X \leq F^{-1}(\alpha / 2)=f_{\alpha / 2} \text { or } X \geq F^{-1}(1-\alpha / 2)=f_{1-\alpha / 2} .
$$

This can be termed a Shewhart chart with asymetric control limits (Chen and Kuo, 2010).

The main work in implementing a chart is implementing functions that compute properties of the chart (e.g. ARL in control, threshold needed to give a certain ARL or hitting probabilities within certain steps). These properties need to be computed given the parameter that is used for running the chart (xi) and given the distribution of the observations $(P)$.

In this example we implement two methods: one for computing the ARL (ARL) and one for computing the $\alpha$ needed to give a certain ARL (calARL). The ARL for the Shewhart chart with asymetric control limits run with estimated parameters will be

$$
\frac{1}{P\left(X \leq \hat{f}_{\alpha / 2}\right)+P\left(X \geq \hat{f}_{1-\alpha / 2}\right)}=\frac{1}{F\left(\hat{f}_{\alpha / 2}\right)+1-F\left(\hat{f}_{1-\alpha / 2}\right)},
$$


and in the implementation below we make a log transform of this to increase the accuracy of the bootstrap. To implement the property calARL, the $\alpha$ needed to achieve a certain ARL is found by a numerical search using the above expression (we have implemented this with ad hoc choices for the boundaries of the numeric search). A logit transform is here used in the implementation to improve the bootstrap.

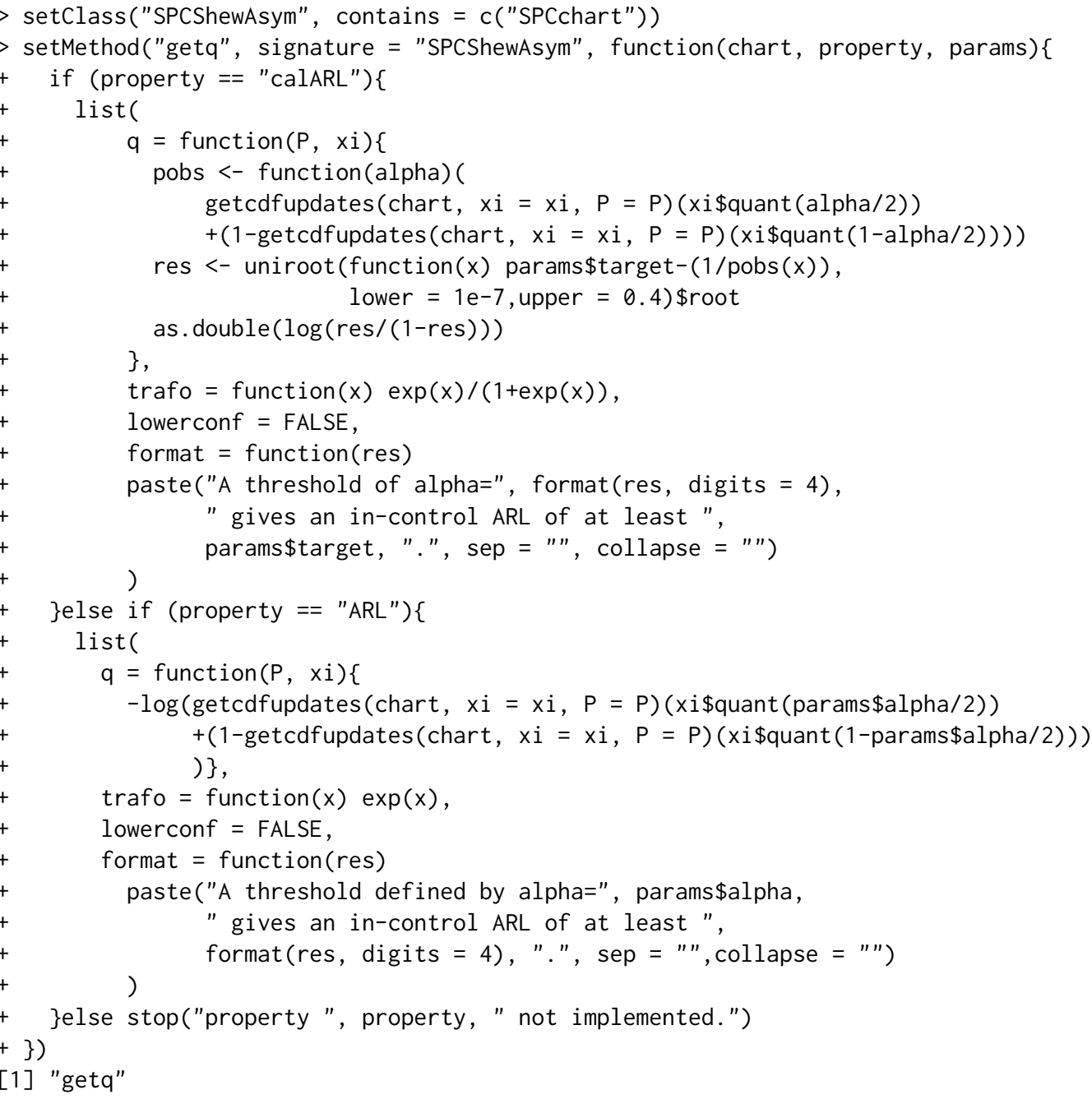

Now we want to use this chart for the example of a gamma distribution. For this we need to implement a basic data model, which uses the observations directly as updates. We estimate the parameter of the gamma distribution via the method of moments (Pofdata). To run the chart we need the quantile function to calculate the estimates of the quantiles $f_{\alpha / 2}$ and $f_{1-\alpha / 2}$ (this appears in xiofP). Resampling is again parametric resampling under the assumed Gamma distribution (resample).

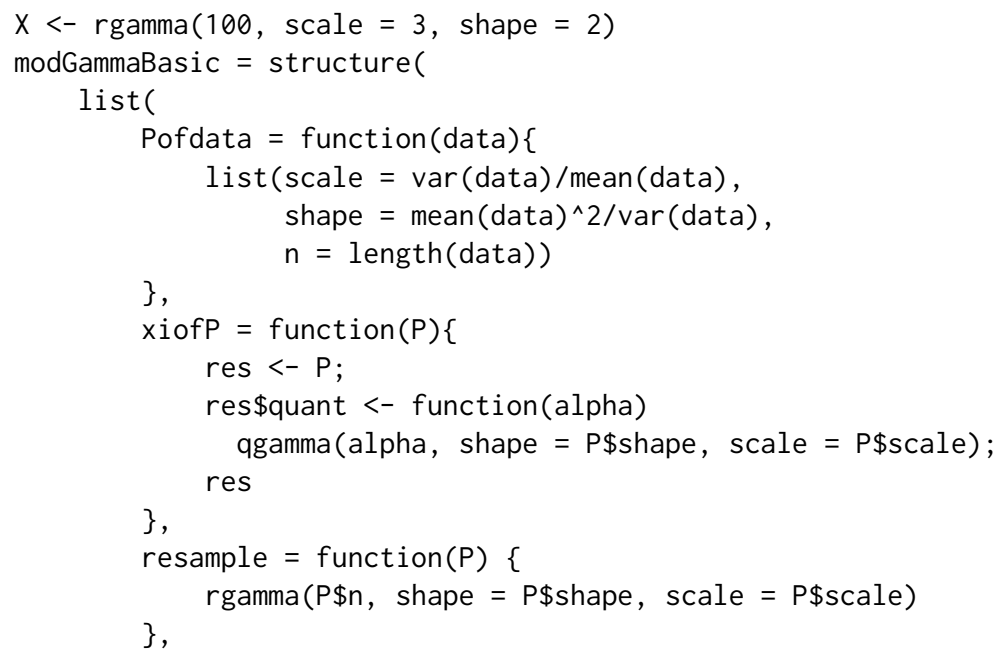




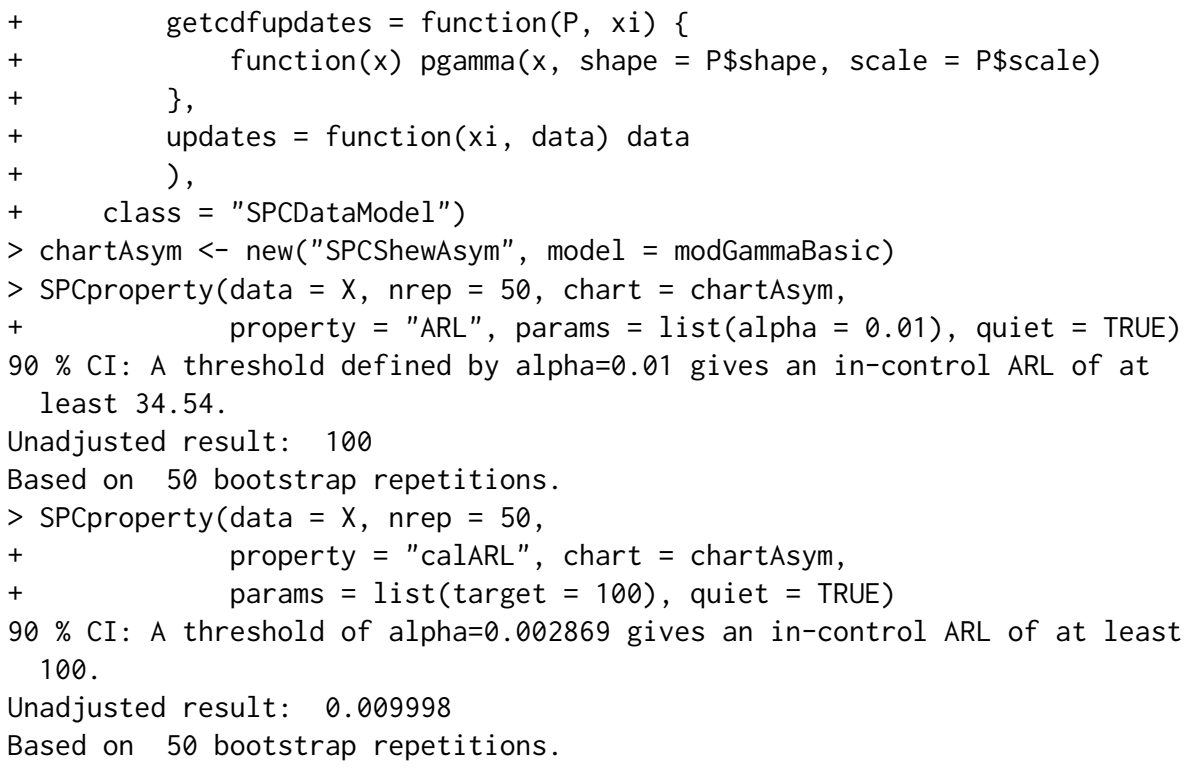

To show the advantage of the modular setup we now modify the data model to assume that the data is coming from an exponential distribution, in other words that the shape parameter of the gamma distribution is 1 . We just need to redefine the function PofData to accomplish this.

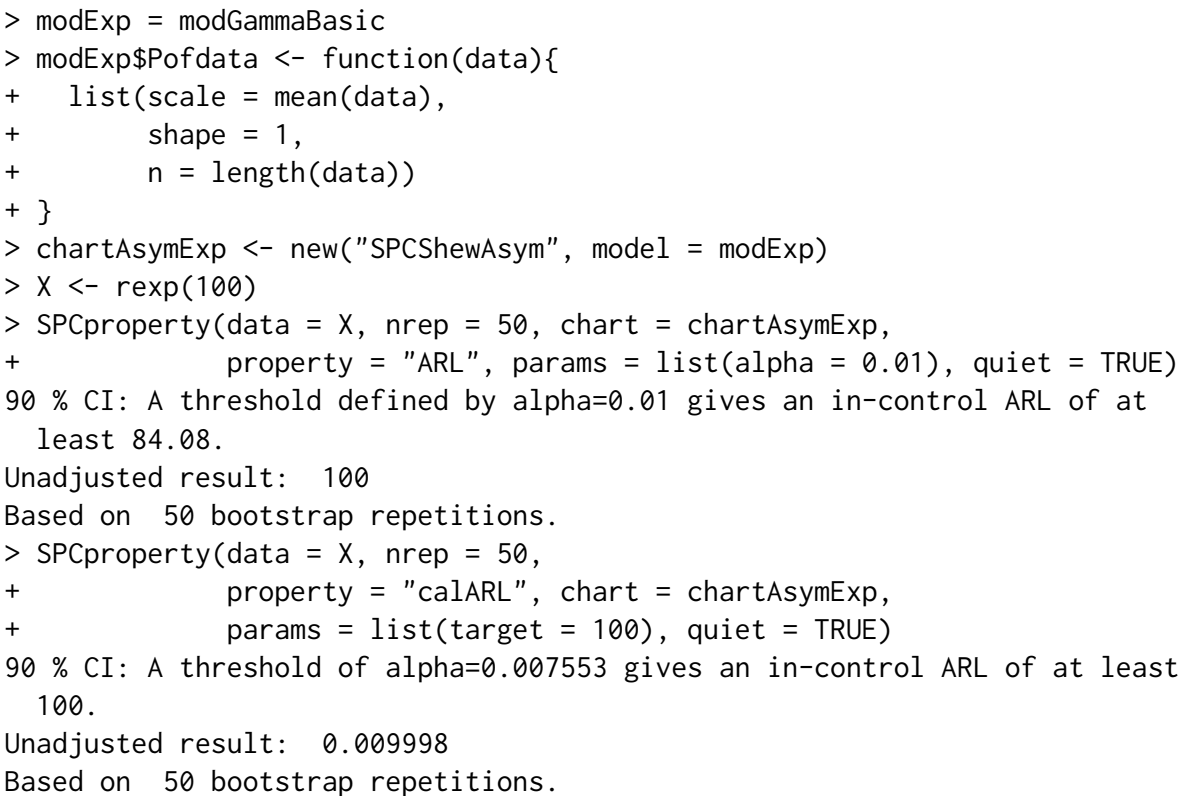

\section{Application to cardiac surgery data}

In this section we illustrate use of the package with an application to a data set on the outcome of cardiac surgery from a UK centre for cardiac surgery over the period 1992-1998. These data were first analysed by Steiner et al. (2000) and have later been used for illustration by several authors (e.g. Sego and Woodall, 2009; Jones and Steiner, 2012; Zhang et al., 2016). A random subset of these data with some random noise added is available in the data frame cardiacsurgery in spcadjust. In this data frame the date of surgery, a surgeon number, the time until death if the patient died during the follow up time and the Parsonnet score of the patient is given. The Parsonnet score is a well established scoring system in cardiac surgery which combines a number of risk factors into a risk score for the patient. The data frame contains 5595 cases.

$>$ data(cardiacsurgery)

Like Steiner et al. (2000) we will focus on the 30-day post-operative mortality rate and use a logistic regression model with Parsonnet score as covariate for taking into account the differences in risk between patients, and use a CUSUM for monitoring. 


\section{CUSUM for logistic regression models}

We first describe the general set up for CUSUM monitoring with logistic regression models and then return to the cardiac surgery example. Assume we have $n$ past in-control data $\left(Y_{-n}, X_{-n}\right), \ldots,\left(Y_{-1}, X_{-1}\right)$, where $Y_{i}$ is a binary response variable and $X_{i}$ is a corresponding vector of covariates. Suppose that in control $\operatorname{logit}\left(\mathrm{P}\left(Y_{i}=1 \mid X_{i}\right)\right)=X_{i} \beta$. A maximum likelihood estimate $\hat{\beta}$ is obtained based on the past data.

For detecting a change to $\operatorname{logit}\left(\mathrm{P}\left(Y_{i}=1 \mid X_{i}\right)\right)=\Delta+X_{i} \beta$, a CUSUM chart based on the cumulative sum of $\log$ likelihood ratios of the out-of-control versus in-control model can be defined by (Steiner et al., 2000)

where

$$
S_{t}=\max \left(0, S_{t-1}+R_{t}\right), \quad S_{0}=0,
$$

$$
\exp \left(R_{t}\right)=\frac{\exp \left(\Delta+X_{t} \beta\right)^{Y_{t}} /\left(1+\exp \left(\Delta+X_{t} \beta\right)\right)}{\exp \left(X_{t} \beta\right)^{Y_{t}} /\left(1+\exp \left(X_{t} \beta\right)\right)}=\exp \left(Y_{t} \Delta\right) \frac{1+\exp \left(X_{t} \beta\right)}{1+\exp \left(\Delta+X_{t} \beta\right)} .
$$

Like in the linear regression case we apply non-parametric bootstrap as described in Section Riskadjusted charts.

\section{Cardiac surgery data}

The two first years of data, containing 1769 cases, are used for estimating the parameters of the logistic regression model. The effect of the Parsonnet score turns out to be non-linear on the logit scale, applying a square root transform of the score sorts out this. We thus set up data for estimating the chart parameters (phase I sample) and for running the chart (phase II sample) as follows:

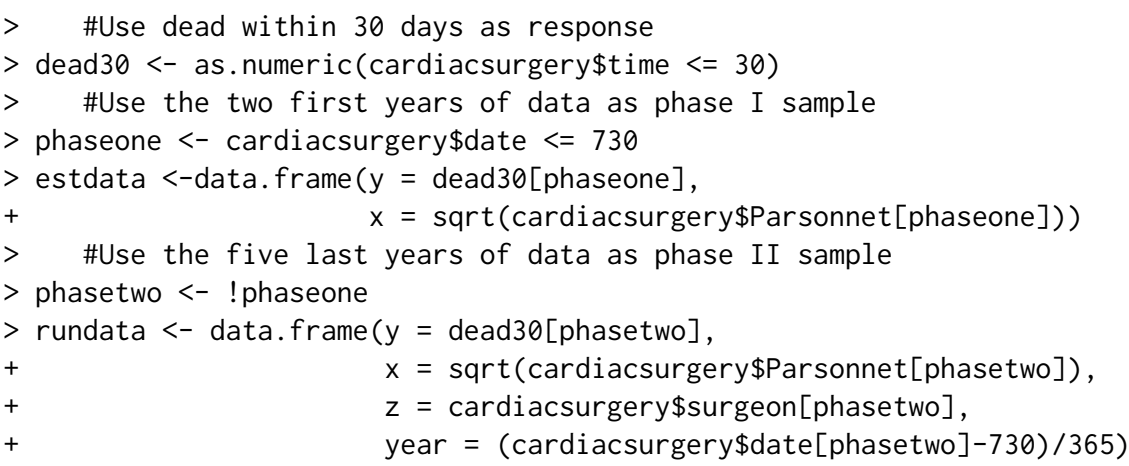

Next we set up charts for monitoring against roughly a doubling and a halving of the mortality rate, respectively. With a baseline rate of $6.1 \%$ this corresponds to $\Delta=0.75$ and $\Delta=-0.75$.

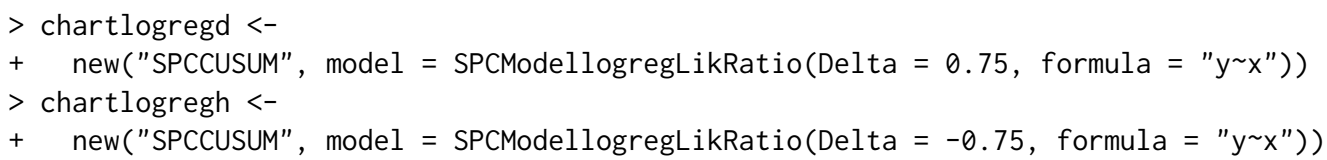

For calculating the thresholds we specify an in control ARL of 10000 , i.e. a false alarm should on average only occur once per 10000 procedures.

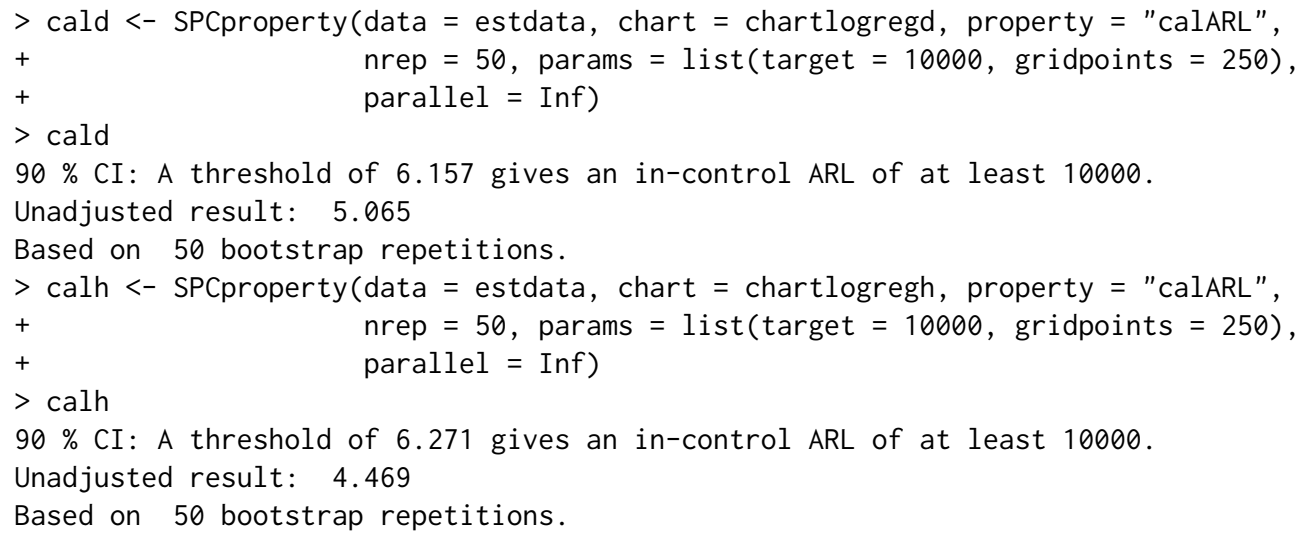

The option parallel = Inf speeds up the bootstrap, but this option must be skipped if the code is run in an environment which does not support parallel processing. 

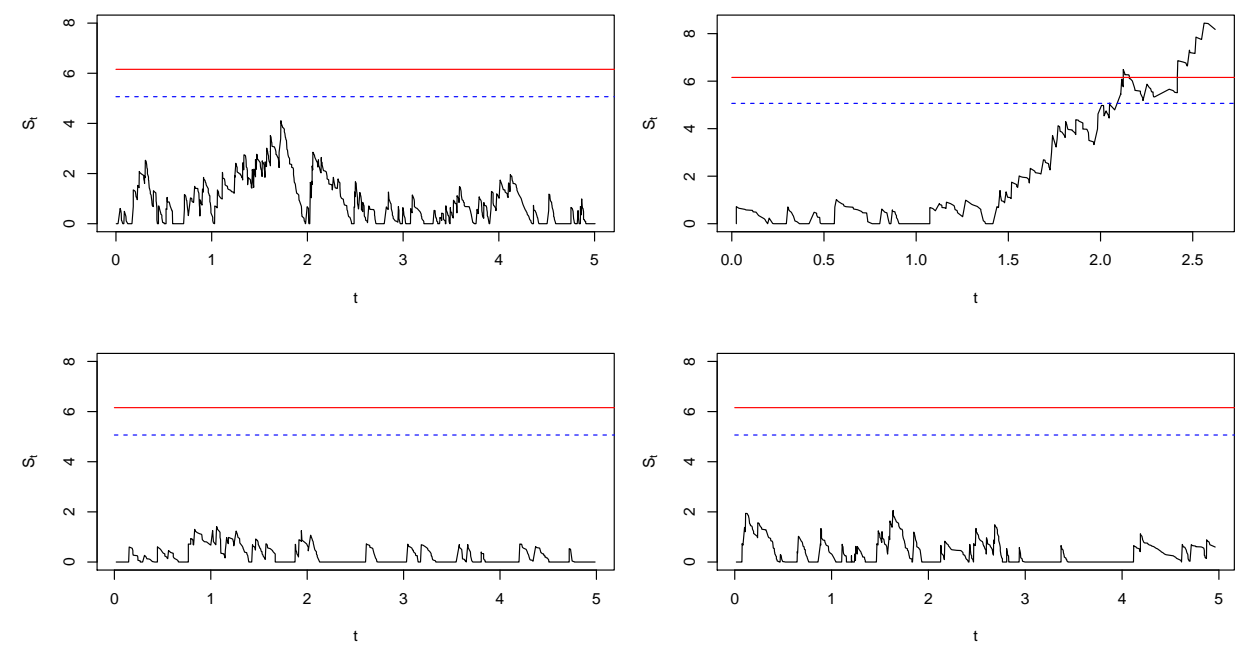

Figure 6: CUSUM charts based on logistic regression model with estimated parameters monitoring against increased mortality. Individual charts for four surgeons.
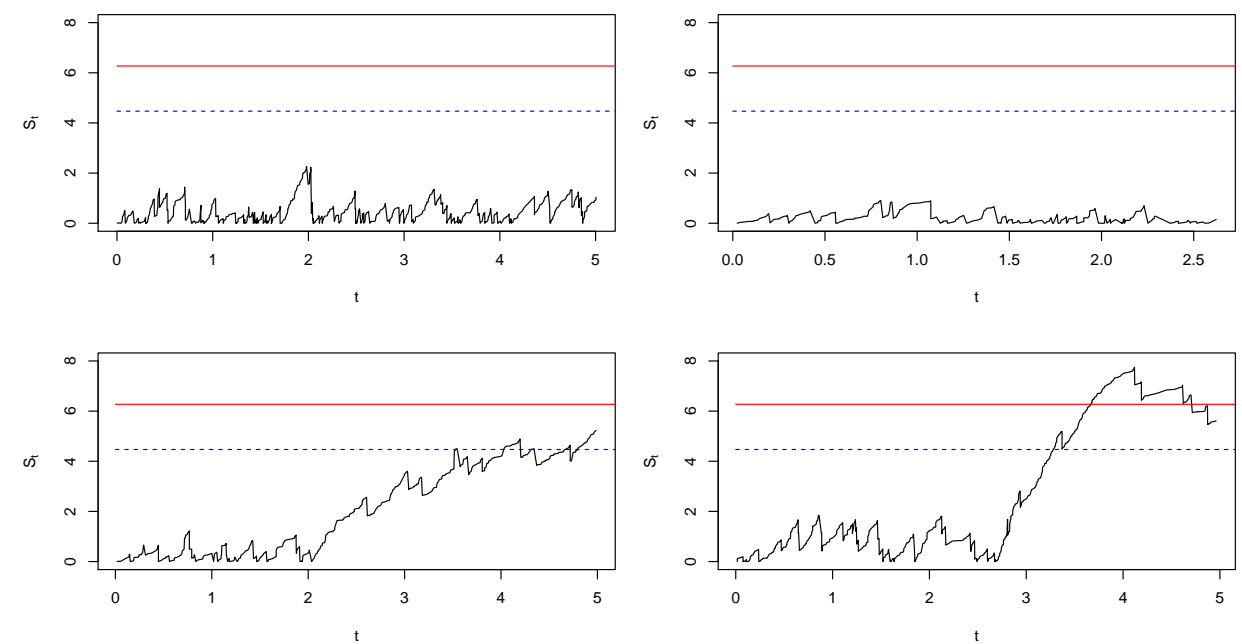

Figure 7: CUSUM charts based on logistic regression model with estimated parameters monitoring against decreased mortality. Individual charts for four surgeons. 
Assuming that the distribution of Parsonnet scores is roughly the same for the patients each surgeon receives, and that the distribution remains approximately the same in the remainder of the period as in the first two years, the thresholds calculated above can be used for running individual charts for each of the surgeons. Notice that in such a setting where several charts are run with the same estimated parameters a threshold adjustment which achieves a guaranteed conditional performance is particularly relevant (Gandy and Kvaløy, 2013).

The resulting CUSUM plots for four of the surgeons are shown in Figures 6 and 7. In Figures 6 the CUSUM for the second surgeon starts to increase after a while and passes both the unadjusted and the adjusted threshold. This could e.g. be due to this surgeon starting to receive more difficult cases, not sufficiently accounted for by the adjustment for Parsonnet score.

For the monitoring against decreased mortality in Figures 7 there is a signal for one of the surgeons, indicating better survival than explained by the adjustment for Parsonnet score. The CUSUM for the third surgeon crosses the unadjusted threshold, but not the adjusted and is thus not regarded as a true signal.

\section{Bibliography}

W. Albers and W. C. M. Kallenberg. Are estimated control charts in control? Statistics, 38:67-79, 2004. URL https://doi.org/10.1080/02669760310001619369. [p458]

W. Albers and W. C. M. Kallenberg. New corrections for old control charts. Quality Engineering, 17: 467-473, 2005. URL https://doi.org/10.1081/QEN-200063498. [p458]

W. Albers, W. C. M. Kallenberg, and S. Nurdiati. Exceedance probabilities for parametric control charts. Statistics, 39:429-443, 2005. URL https://doi .org/10.1080/02331880500310181. [p458]

P. Biswas and J. D. Kalbfleisch. A risk-adjusted CUSUM in continuous time based on the Cox model. Statistics in Medicine, 27:3382-3406, 2008. URL https://doi .org/10.1002/sim. 3216. [p458]

A. Bottle and P. Aylin. Intelligent information: a national system for monitoring clinical performance. Health Services Research, 43:10-31, 2008. URL https://doi .org/10.1111/j.1475-6773.2007.00742. x. [p458]

G. Capizzi and G. Masarotto. Bootstrap-based design of residual control charts. IIE Transactions, 41: 275-286, 2009. URL https://doi .org/10.1080/07408170802120059. [p458]

C. W. Champ and L. A. Jones-Farmer. Properties of multivariate control charts with estimated parameters. Sequential Analysis, 26:153-169, 2007. URL https://doi .org/10.1080/07474940701247040. [p458]

S. Chatterjee and P. Qiu. Distribution-free cumulative sum control charts using bootstrap-based control limits. Annals of Applied Statistics, 3:349-369, 2009. URL https://doi .org/10.1214/08-A0AS197. [p458]

H. Chen and W.-L. Kuo. Comparison of the symmetric and asymmetric control limits for $\bar{X}$ and $R$ charts. Computers and Industrial Engineering, 59:903-910, 2010. URL https://doi . org/10.1016/j. cie.2010.08.021. [p469]

M. Flores. Qcr: Quality Control Review, 2016. URL https://CRAN. R-project.org/web/package=qcr. R package version 1.0. [p458]

M. Fouladirad, A. Grall, and L. Dieulle. On the use of on-line detection for maintenance of gradually deteriorating systems. Reliability Engineering and System Safety, 93:1814-1820, 2008. URL https: //doi.org/10.1016/j.ress.2008.03.020. [p458]

M. Frisén, editor. Financial Surveillance. John Wiley \& Sons, 2008. [p458]

A. Gandy and J. T. Kvaløy. Guaranteed conditional performance of control charts via bootstrap methods. Scandinavian Journal of Statistics, 40:647-668, 2013. URL https: //doi . org/10. 1002/sjos . 12006. [p458, 460, 461, 462, 474]

A. Gandy and J. T. Kvaløy. Spcadjust: Functions for Calibrating Control Charts, 2016. URL https: //CRAN.R-project.org/web/package=spcadjust. R package version 1.1. [p458]

A. Gandy, J. T. Kvaløy, A. Bottle, and F. Zhou. Risk-adjusted monitoring of time to event. Biometrika, 97:375-388, 2010. URL https://doi.org/10.1093/biomet/asq004. [p458] 
O. Grigg and V. Farewell. An overview of risk-adjusted charts. Journal of the Royal Statistical Society A, 167:523-539, 2004. URL https://doi.org/10.1111/j.1467-985X.2004.0apm2.x. [p458, 461]

D. M. Hawkins and D. H. Olwell. Cumulative Sum Charts and Charting for Quality Improvement. SpringerVerlag, 1998. [p468]

L. Horváth, M. H. a, P. Kokoszka, and J. Steinebach. Monitoring changes in linear models. Journal of Statistical Planning and Inference, 126:225-251, 2004. URL https://doi .org/10.1016/ j. jspi. 2003. 07. 014. [p461]

W. A. Jensen, L. A. Jones-Farmer, C. W. Champ, and W. H. Woodall. Effects of parameter estimation on control chart properties: A literature review. Journal of Quality Technology, 38:349-364, 2006. [p458]

L. A. Jones. The statistical design of EWMA control charts with estimated parameters. Journal of Quality Technology, 34:277-288, 2002. [p458]

L. A. Jones, C. W. Champ, and S. E. Rigdon. The run length distribution of the CUSUM with estimated parameters. Journal of Quality Technology, 36:95-108, 2004. [p458]

M. A. Jones and S. H. Steiner. Assessing the effect of estimation error on risk-adjusted CUSUM chart performance. International Journal for Quality in Health Care, 24:176-181, 2012. URL https: //doi.org/10.1093/intahc/mzr082. [p458, 471]

S. Knot. Spc: Statistical Process Control - Collection of Some Useful Functions, 2016. URL https: //CRAN. Rproject. org/web/package=spc. R package version 0.5.3. [p458]

N. A. Saleh, M. A. Mahmoud, M. J. Keefe, and W. H. Woodall. The difficulty in designing Shewhart $\bar{X}$ and X control charts with estimated parameters. Journal of Quality Technology, 47:127-138, 2015. [p458]

M. Salmon, D. Schumacher, and M. Höhle. Monitoring count time series in R: Aberration detection in public health surveillance. Journal of Statistical Software, 70(10):1-35, 2016. URL https: //doi .org/ 10.18637/jss.v070.i10. [p458]

E. Santos-Fernández. Multivariate Statistical Quality Control Using R, volume 14. Springer-Verlag, 2013. ISBN 9781461454533. URL https://doi.org/10.1007/978-1-4614-5453-3. [p458]

W. Schmid, editor. 80 Years of the Control Chart. Sequential Analysis, 26(2), 2007a. [p458]

W. Schmid, editor. 80 Years of the Control Chart. Sequential Analysis, 26(3), 2007b. [p458]

L. Scrucca. Qcc: An R package for quality control charting and statistical process control. R News, 4: 11-17, 2004. [p458]

L. Scrucca. IQCC: Improved Quality Control Charts, 2014. URL https://CRAN. R-project. org/web/ package=IQCC. R package version 0.6. [p458]

L. H. Sego and W. H. Woodall. Risk-adjusted monitoring of survival times. Statistics in Medicine, 28: 1386-1401, 2009. URL https://doi.org/10.1002/sim.3546. [p471]

S. H. Steiner, R. J. Cook, V. T. Farewell, and T. Treasure. Monitoring surgical performance using risk-adjusted cumulative sum charts. Biostatistics, 1:441-452, 2000. URL https://doi .org/10.1093/ biostatistics/1.4.441. [p471,472]

Z. G. Stoumbos, M. R. Reynolds Jr., Ryan, T. P., and W. H. Woodall. The state of statistical process control as we proceed into the 21st century. Journal of the American Statistical Association, 95:992-998, 2000. URL https://doi.org/10.1080/01621459.2000.10474292. [p458]

W. H. Woodall. The use of control charts in health-care and public-health surveillance. Journal of Quality Technology, 38:89-134, 2006. With discussion. [p458]

M. Zhang, Y. Xu, Z. He, and X. Hou. The effect of estimation error on risk-adjusted survival time CUSUM chart performance. Quality and Reliability Engineering International, 32:1445-1452, 2016. URL https://doi.org/10.1002/qre.1849. [p458,471]

Y. Zhang, P. Castagliola, Z. Wu, and M. B. C. Khoo. The synthetic $\bar{X}$ chart with estimated parameters. IIE Transactions, 43:676-687, 2011. URL https://doi.org/10.1080/0740817X.2010.549547. [p458]

W. Zhu and C. Park. edcc: An R package for the economic design of the control chart. Journal of Statistical Software, 52(9):1-24, 2013. URL https://doi.org/10. 18637/jss. v052.i09. [p458] 
Axel Gandy

Department of Mathematics

Imperial College London

London, SW7 2AZ, UK

a.gandy@imperial.ac.uk

Jan Terje Kvaløy

Department of Mathematics and Natural Sciences

University of Stavanger

4036 Stavanger, Norway

jan.t.kvaloy@uis.no 